\title{
Non-Hermitian multichannel theory of ultracold collisions modified by intense light fields tuned to the red of the trapping transition
}

\author{
R. Napolitano \\ Instituto de Química de Araraquara, Universidade Estadual Paulista, Araraquara, São Paulo 14800-900, Brazil \\ and Instituto de Física de São Carlos, Universidade de São Paulo, São Carlos, São Paulo 13560-970, Brazil
}

(Received 15 August 1997)

\begin{abstract}
We develop a systematic scheme to treat binary collisions between ultracold atoms in the presence of a strong laser field, tuned to the red of the trapping transition. We assume that the Rabi frequency is much less than the spacing between adjacent bound-state resonances. In this approach we neglect fine and hyperfine structures, but consider fully the three-dimensional aspects of the scattering process, up to the partial $d$ wave. We apply the scheme to calculate the $S$ matrix elements up to the second order in the ratio between the Rabi frequency and the laser detuning. We also obtain, for this simplified multichannel model, the asymmetric line shapes of photoassociation spectroscopy, and the modification of the scattering length due to the light field at low, but finite, entrance kinetic energy. We emphasize that the present calculations can be generalized to treat more realistic models, and suggest how to carry out a thorough numerical comparison to this semianalytic theory. [S1050-2947(98)04902-6]
\end{abstract}

PACS number(s): 32.80.Pj, 34.50.-s, 34.50.Rk

\section{INTRODUCTION}

We can modify and control certain aspects of ultracold collisions using laser fields suitably tuned and polarized. Indeed, recent experimental [1-8] and theoretical [9-11] studies have assured our ability to suppress inelastic ultracoldcollision rates by light fields tuned to the blue of the trapping transition. Moreover, in the context of quantum degeneracy, a zero-temperature calculation [12] has shown the possibility of modifying the scattering length associated with the interaction potential of two ground-state atoms, in the presence of red-detuned photons.

Recent experiments show that Bose-Einstein condensation of trapped alkali-metal atoms occurs at finite ultracold temperatures [13-15]. In this energy regime, we have developed a two-state model for controlling scattering lengths and photoassociation spectral line shapes of alkali-metal atoms by red-detuned laser fields [16]. However, at high enough intensities of the light field, a two-state model cannot describe the collision completely. Due to multiphoton processes, partial waves higher than the $s$ wave contribute appreciably to the scattering [17]. Furthermore, atom-field dressing effects manifest themselves when the collision occurs in the presence of a strong enough laser field. Below 1 $\mu \mathrm{K}$, light shifts become comparable to the entrance collision energies, even for detunings of several tens $\mathrm{cm}^{-1}$ from the trapping transition. We expect dressing effects to be especially relevant to photoassociation spectral line shapes of alkali-metal atoms, since this topic has been extensively studied in the past few years mostly in the low-intensity regime [18-26].

In this paper we present a systematic approach to treat binary ultracold collisions in the presence of an intense laser field, tuned to the red of the trapping transition. The only assumption we make concerning the light intensity is that the corresponding Rabi frequency is much less than the spacing between adjacent bound-state resonances. We predict, in a unified way, the modification of scattering lengths and photoassociation spectral line shapes, regarding only isolated resonances. With this simple model we are able to take into account multichannel and dressing effects in the regime of finite ultracold temperatures. As in Ref. [10], we take a step beyond the two-state approaches by neglecting fine and hyperfine structures, but considering fully the threedimensional aspects of the problem. For simplicity and concreteness, we introduce partial waves up to the $d$ wave and treat the particular case of a pair of sodium atoms in the presence of linearly polarized, red-detuned photons. In a forthcoming paper we will present numerical calculations based on the present theory and a comparison between the effects of linear and circular polarizations. We emphasize that the present approach is easily extensible to higher partial waves and other alkali-metal species. This treatment can also accommodate more than one ground-state potential curve and retardation effects. Although it may be considered cumbersome, fine and hyperfine structures can, in principle, also be incorporated into the formalism.

The solution of the coupled equations describing the collision process is fully detailed in this paper, in a selfcontained manner, for completeness and clarity. Although we present a systematic algorithm to calculate dressing effects to all orders, we illustrate the procedure through explicit calculations for a special situation relevant to sodium, up to the second order in the ratio between the Rabi frequency and the detuning. In the present treatment we intend to emphasize multichannel, finite-temperature, and dressing effects, without unnecessary sophistication.

To account for spontaneous emission from the excited bound state, we adopt a non-Hermitian effective Hamiltonian that we describe in detail in Sec. II. Following this specification of the problem in the undressed basis, including all the coupled states up to the partial $d$ wave, in Sec. III we reformulate the collision process in terms of the asymptotic dressed states. In the dressed basis, the Hamiltonian is asymptotically diagonal and this basis represents the actual 
incoming and outgoing physical states. In Sec. IV we consider the closed-channel manifold assuming that it contains an isolated resonance. In this way we simplify the closedchannel problem by regarding only the resonant contribution to the corresponding Green function. We obtain the $S$ matrix elements in Sec. V, up to the second order in the ratio between the Rabi frequency and the detuning. We also illustrate the application of the procedure by calculating the asymmetric line shapes of photoassociation spectroscopy, and the modification of the scattering length by the light field. In Sec. VI we discuss the region of parameter space where we expect this theory to be valid. Finally, in Sec. VII we conclude by summarizing the present approach and presenting some directions we will follow in a forthcoming paper.

\section{THE UNDRESSED POTENTIAL MATRIX}

In this article we consider two sodium atoms in the hyperfine state $\left|F=1, m_{F}=-1\right\rangle$, as in Ref. [15], so that the lower molecular state involved is the $a^{3} \Sigma_{u}^{+}$. From this lower molecular state, the colliding system can absorb a reddetuned photon and be excited to a bound state of symmetry ${ }^{3} \Sigma_{g}^{+}$. Asymptotically, that is, when the interatomic distance $R$ approaches a large but finite value $R_{\infty}$ at which we can neglect the interaction between the atoms, the $a^{3} \Sigma_{u}^{+}$molecular state corresponds to two atoms in their ${ }^{2} S$ ground states. The ${ }^{3} \Sigma_{g}^{+}$bound molecule asymptotically dissociates into one atom in its ${ }^{2} S$ ground state and another in its ${ }^{2} P$ first excited state. Because in this paper we ignore fine and hyperfine interactions, let us denote by $j$ the electronic orbital angular-momentum quantum number of the asymptotic two-atom system. Thus, if the two atoms are in their ${ }^{2} S$ ground states, then $j=0$; and if one of the atoms is in its ${ }^{2} S$ ground state and the other in its ${ }^{2} P$ first excited state, then $j=1$. The $j=0$ state has angular-momentum projection $m_{j}$ $=0$ along a space-fixed $z$ axis, while the $j=1$ state may have $m_{j}=-1,0$, or +1 . Therefore, the asymptotic electronic basis states relevant to this problem are denoted $\left|j, m_{j}\right\rangle$, with $m_{j}=0$ for $j=0$ and $m_{j}=0, \pm 1$ for $j=1$.

Two separated atoms can come together at different impact parameters. Equivalently, we can describe the rotational state of the approaching atoms by introducing the spherical harmonic functions $\left|l, m_{l}\right\rangle \equiv Y_{l}^{m_{l}}(\theta, \varphi)$, where $l=0,2,4, \ldots$ for bosons [27] and $m_{l}=-l,-l+1, \ldots, l-1, l$. The angles $\theta$ and $\varphi$ describe the orientation of the interatomic axis with respect to the space-fixed reference frame. Up to the $l=2$ partial wave ( $d$ wave) and for linear light-field polarization, the only coupled undressed states, including the ground state, are given by [10]

$$
\begin{gathered}
|1\rangle \equiv\left|j=0, m_{j}=0\right\rangle\left|l=0, m_{l}=0\right\rangle|N\rangle, \\
|2\rangle \equiv\left|j=0, m_{j}=0\right\rangle\left|l=2, m_{l}=0\right\rangle|N\rangle, \\
|3\rangle \equiv\left|j=1, m_{j}=0\right\rangle\left|l=0, m_{l}=0\right\rangle|N-1\rangle, \\
|4\rangle \equiv\left|j=1, m_{j}=-1\right\rangle\left|l=2, m_{l}=1\right\rangle|N-1\rangle, \\
|5\rangle \equiv\left|j=1, m_{j}=1\right\rangle\left|l=2, m_{l}=-1\right\rangle|N-1\rangle,
\end{gathered}
$$

$$
|6\rangle \equiv\left|j=1, m_{j}=0\right\rangle\left|l=2, m_{l}=0\right\rangle|N-1\rangle,
$$

and we have also included the radiation-field Fock states $|N\rangle$ and $|N-1\rangle$ containing $N$ and $(N-1)$ photons, respectively. These states are normalized to unity, that is, $\langle\alpha \mid \beta\rangle=\delta_{\alpha, \beta}$, for $\alpha, \beta=1, \ldots, 6$. The radiation can only couple $|1\rangle$ to $|3\rangle$ and $|2\rangle$ to $|6\rangle$, but there are other couplings that force the introduction of the states $|4\rangle$ and $|5\rangle$, as we show below. The radiative couplings we consider are

$$
\left\langle 1\left|H_{I}\right| 3\right\rangle=\left\langle 2\left|H_{I}\right| 6\right\rangle=\hbar \Omega,
$$

where $\Omega=\sqrt{2} \Omega_{A}$ and $\Omega_{A}$ is the atomic Rabi frequency for the $3{ }^{2} S \rightarrow 3{ }^{2} P$ transition of sodium given by $\Omega_{A} /(2 \pi c)$ $\approx 1.47966 \times 10^{-3} \sqrt{I} \mathrm{~cm}^{-1}$, and $I$ is the laser intensity in $\mathrm{W} / \mathrm{cm}^{2}[10]$. Let us notice that the molecular Rabi frequency $\Omega$ is a real quantity, independent of $R$.

The total Hamiltonian for this problem has the form

$$
H=-\frac{\hbar^{2}}{2 \mu R} \frac{\partial^{2}}{\partial R^{2}} R+\frac{\mathbf{L}^{2}}{2 \mu R^{2}}+V(R),
$$

with the potential operator

$$
V(R)=H_{e}(R)+H_{f}+H_{I}+\mathbf{1} \xi,
$$

where $\mathbf{L} \equiv-i \hbar \mathbf{R} \times \nabla$ is the rotational angular-momentum operator satisfying $\quad \mathbf{L}^{2}\left|l, m_{l}\right\rangle=\hbar^{2} l(l+1)\left|l, m_{l}\right\rangle \quad$ and $\hat{\mathbf{z}} \cdot \mathbf{L}\left|l, m_{l}\right\rangle=\hbar m_{l}\left|l, m_{l}\right\rangle, \mathbf{R}$ is the interatomic vector of polar coordinates $(R, \theta, \varphi), \mu$ is the reduced mass of the colliding partners, $H_{e}(R)$ is the electronic Hamiltonian for a fixed interatomic separation $R, H_{f}$ is the radiation-field Hamiltonian, $H_{I}$ is the operator representing the interaction between the colliding atoms and the electromagnetic field, $\mathbf{1}$ is the identity operator, and $\xi$ is an arbitrary shift in energy we specify below. The nonzero matrix elements of $H_{I}$ are given by Eq. (2). The action of $H_{f}$ on a Fock state containing $N$ photons of frequency $\omega$ is

$$
H_{f}|N\rangle=\hbar \omega\left(N+\frac{1}{2}\right)|N\rangle .
$$

Let $E_{0}$ be the total electronic energy (not including atomic kinetic energy) of two separated ${ }^{2} S$ atoms and $E_{1}$ the total electronic energy of a ${ }^{2} S$ atom and a distant ${ }^{2} P$ atom. Hence, $H_{e}(R)$ is diagonal in the basis set $\left\{\left|j, \Lambda, J, M_{J}\right\rangle\right\}$ :

$$
H_{e}(R)\left|j, \Lambda, J, M_{J}\right\rangle=\left[W_{j_{\Lambda \mid}}(R)+E_{j}-i \hbar \gamma \delta_{1, j}\right]\left|j, \Lambda, J, M_{J}\right\rangle,
$$

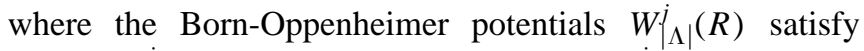
$\lim _{R \rightarrow \infty} W \dot{j}_{\Lambda \mid}(R)=0$ and $R_{\infty}$ is such that $W j_{\Lambda \mid}^{j}\left(R \approx R_{\infty}\right) \approx 0$, and the Hund's-case (a) eigenfunctions $\left|j, \Lambda, J, M_{J}\right\rangle$ are defined as

$$
\left|j, \Lambda, J, M_{J}\right\rangle \equiv \sqrt{\frac{2 J+1}{4 \pi}} D_{M_{J}, \Lambda}^{J *}(\varphi, \theta, 0)|j, \Lambda\rangle
$$

and

$$
|j, \Lambda\rangle \equiv \sum_{m_{j}=-1}^{1} D_{\Lambda, m_{j}}^{j *}(\varphi, \theta, 0)\left|j, m_{j}\right\rangle .
$$

Here, $\hbar \gamma$ is the atomic linewidth (the molecular linewidth is equal to $2 \hbar \gamma$ [28]) of the $3{ }^{2} P$ state of sodium that we in- 
troduce at this point in an $a d h o c$ fashion to account for the spontaneous emission from the bound-state resonance $[16,29]$. The unnormalized symmetric top eigenfunctions $D_{M_{J}, \Lambda}^{J *}(\varphi, \theta, 0)$ and $D_{\Lambda, m_{j}}^{j *}(\varphi, \theta, 0)$ are the Wigner rotation matrix elements [30]. The functions $|j, \Lambda\rangle$ refer to a molecule-fixed reference frame that has its $z$ axis parallel to R. Let us notice that $\Lambda=0$ for $j=0$ and $\Lambda=0, \pm 1$ for $j$ $=1$, given the isotropy of space. As explained in Ref. [10], the implicit $R$ dependence of the molecular eigenfunctions $\left|j, \Lambda, J, M_{J}\right\rangle$ is of no appreciable effect due to the smallness of the ratio between the electronic and nuclear mass (BornOppenheimer approximation). The meaning of the quantum numbers $J$ and $M_{J}$ is clear from the equations $\mathbf{J} \equiv \mathbf{j}+\mathbf{L}$, $\mathbf{J}^{2}\left|j, \Lambda, J, M_{J}\right\rangle=\hbar^{2} J(J+1)\left|j, \Lambda, J, M_{J}\right\rangle$, and $\hat{\mathbf{z}} \cdot \mathbf{J}\left|j, \Lambda, J, M_{J}\right\rangle$ $=\hbar M_{J}\left|j, \Lambda, J, M_{J}\right\rangle$, with $J=|j-l|,|j-l|+1, \ldots, j+l-1$, $j+l$ and $M_{J}=-J,-J+1, \ldots, J-1, J$.

$$
V(R)=\left[\begin{array}{ccc}
W_{0}^{0}(R) & 0 & \hbar \Omega \\
0 & W_{0}^{0}(R) & 0 \\
\hbar \Omega & 0 & V_{a}(R)-\hbar \Delta_{c} \\
0 & 0 & D(R) / \sqrt{15} \\
0 & 0 & D(R) / \sqrt{15} \\
0 & \hbar \Omega & -2 \sqrt{5} D(R) / 15
\end{array}\right.
$$

where $\Delta_{c} \equiv \Delta+i \gamma$ is a complex quantity, $\Delta \equiv \omega-\left(E_{1}\right.$ $\left.-E_{0}\right) / \hbar<0$ is the laser detuning that we assume to be negative (red detuning), and we have defined the functions

$$
\begin{gathered}
D(R) \equiv W_{1}^{1}(R)-W_{0}^{1}(R), \\
V_{a}(R) \equiv \frac{2 W_{1}^{1}(R)+W_{0}^{1}(R)}{3}, \\
V_{b}(R) \equiv \frac{5 W_{1}^{1}(R)+2 W_{0}^{1}(R)}{7}, \\
V_{c}(R) \equiv \frac{10 W_{1}^{1}(R)+11 W_{0}^{1}(R)}{21} .
\end{gathered}
$$

To derive this potential matrix we have chosen the energy shift appearing in Eq. (4) to be $\xi \equiv-E_{0}-\hbar \omega\left(N+\frac{1}{2}\right)$, for the sake of simplicity.

\section{THE CLOSE-COUPLING FORMULATION IN THE DRESSED-STATE REPRESENTATION}

In the close-coupling formalism, described in detail in Ref. [10] and references cited therein, we look for wave functions, written as

$$
\left|\Psi_{\beta}(\mathbf{R})\right\rangle=\sum_{\sigma=1}^{6} \frac{F_{\sigma, \beta}(R)}{R}|\sigma\rangle,
$$

The Born-Oppenheimer molecular basis $\left\{\left|j, \Lambda, J, M_{J}\right\rangle\right\}$ is related to the asymptotic space-fixed basis $\left\{\left|j, m_{j}\right\rangle\left|l, m_{l}\right\rangle\right\}$ through the relation [31]

$$
\begin{aligned}
\left|j, m_{j}\right\rangle\left|l, m_{l}\right\rangle= & \sum_{J=|j-l|}^{j+l} \sum_{M_{J}=-J}^{J} \sum_{\Lambda=-j}^{j} \sqrt{\frac{2 l+1}{2 J+1}} \\
& \times\left\langle j, l, m_{j}, m_{l} \mid J, M_{J}\right\rangle \\
& \times\langle j, l, \Lambda, 0 \mid J, \Lambda\rangle\left|j, \Lambda, J, M_{J}\right\rangle,
\end{aligned}
$$

adopting the notation of Ref. [30] to write Clebsch-Gordan coefficients as, for example, $\left\langle j, l, m_{j}, m_{l} \mid J, M_{J}\right\rangle$. In the undressed basis, Eqs. (1), the potential operator of Eq. (4) can be represented by a $6 \times 6$ complex matrix through the use of Eqs. (2), (5)-(8):

$$
\left.\begin{array}{ccc}
0 & 0 & 0 \\
0 & 0 & \hbar \Omega \\
D(R) / \sqrt{15} & D(R) / \sqrt{15} & -2 \sqrt{5} D(R) / 15 \\
V_{b}(R)-\hbar \Delta_{c} & -2 D(R) / 7 & \sqrt{3} D(R) / 21 \\
-2 D(R) / 7 & V_{b}(R)-\hbar \Delta_{c} & \sqrt{3} D(R) / 21 \\
\sqrt{3} D(R) / 21 & \sqrt{3} D(R) / 21 & V_{c}(R)-\hbar \Delta_{c}
\end{array}\right],
$$

that satisfy the time-independent Schrödinger equation, $H\left|\Psi_{\beta}(\mathbf{R})\right\rangle=E\left|\Psi_{\beta}(\mathbf{R})\right\rangle$, where $H$ is the total Hamiltonian given by Eq. (3), $E$ is an eigenvalue corresponding to the continuous spectrum of $H$, and the index $\beta$ enumerates the eigenstates belonging to a given $E$. The eigenvalue $E$ of $H$ is a complex quantity, since $H$ is not Hermitian, and in the following we show that the suitable choice of asymptotic boundary conditions implies a finite imaginary part of $E$. It then follows that the solution of the time-dependent Schrödinger equation, $H\left|\Phi_{\beta}(\mathbf{R}, t)\right\rangle=i \hbar(\partial / \partial t)\left|\Phi_{\beta}(\mathbf{R}, t)\right\rangle$, is given by $\left|\Phi_{\beta}(\mathbf{R}, t)\right\rangle=\exp [-i \operatorname{Re}(E) t / \hbar+\operatorname{Im}(E) t / \hbar]\left|\Psi_{\beta}(\mathbf{R})\right\rangle$. Because $\operatorname{Im}(E)<0$, the amplitude of the state $\left|\Phi_{\beta}(\mathbf{R}, t)\right\rangle$ decreases exponentially, due to spontaneous emission. In this section we also show that the magnitude of $\operatorname{Im}(E)$ can be made very small by choosing a sufficiently large red detuning.

Substituting Eqs. (3) and (11) into the time-independent Schrödinger equation gives a matrix equation for the radial amplitudes $F_{\sigma, \beta}(R)$ :

$$
-\frac{\hbar^{2}}{2 \mu} \frac{d^{2}}{d R^{2}} F_{\beta}(R)+\frac{\mathbf{L}^{2}}{2 \mu R^{2}} F_{\beta}(R)+V(R) F_{\beta}(R)=E F_{\beta}(R),
$$

where

$$
F_{\beta}(R) \equiv\left[F_{1, \beta}(R) F_{2, \beta}(R) F_{3, \beta}(R) F_{4, \beta}(R) F_{5, \beta}(R) F_{6, \beta}(R)\right]^{t}
$$

is a $6 \times 1$ column matrix (the superscript $t$ indicates matrix 
transposing), $V(R)$ is the $6 \times 6$ complex potential matrix of Eq. (9), and $\mathbf{L}^{2}$ is a $6 \times 6$ diagonal matrix containing, along its diagonal, the sequence of eigenvalues of the square of the rotational angular-momentum operator, ordered according to the basis set of Eqs. (1): $\left(0,6 \hbar^{2}, 0,6 \hbar^{2}, 6 \hbar^{2}, 6 \hbar^{2}\right)$. Asymptotically, Eq. (12) becomes $\hbar^{2} d^{2} F_{\beta}(R) / d R^{2}-2 \mu V_{\infty} F_{\beta}(R)=0$, where $V_{\infty}$ is a $6 \times 6 R$-independent complex matrix defined as

$$
V_{\infty} \equiv\left[\begin{array}{cccccc}
-E & 0 & \hbar \Omega & 0 & 0 & 0 \\
0 & -E & 0 & 0 & 0 & \hbar \Omega \\
\hbar \Omega & 0 & -\hbar \Delta_{c}-E & 0 & 0 & 0 \\
0 & 0 & 0 & -\hbar \Delta_{c}-E & 0 & 0 \\
0 & 0 & 0 & 0 & -\hbar \Delta_{c}-E & 0 \\
0 & \hbar \Omega & 0 & 0 & 0 & -\hbar \Delta_{c}-E
\end{array}\right] .
$$

The matrix $V_{\infty}$ is not diagonal at very large interatomic separation, meaning that the coupling with the radiation is always present. Therefore, the undressed states, Eqs. (1), are not suitable for describing the asymptotic states necessary to define the $S$ matrix. In other words, in the actual physical situation of a collision in the presence of photons, the incoming and outgoing scattering states are the stationary states of the asymptotic Hamiltonian. Accordingly, the normalized eigenstates of $V_{\infty}$ (the dressed states) are obtained by diagonalizing Eq. (13) and are given by

$$
\begin{gathered}
\left|D_{1}\right\rangle \equiv a_{-}|1\rangle+b_{-}|3\rangle, \\
\left|D_{2}\right\rangle \equiv a_{-}|2\rangle+b_{-}|6\rangle, \\
\left|D_{3}\right\rangle \equiv a_{+}|1\rangle+b_{+}|3\rangle, \\
\left|D_{4}\right\rangle \equiv|4\rangle, \\
\left|D_{5}\right\rangle \equiv|5\rangle, \\
\left|D_{6}\right\rangle \equiv a_{+}|2\rangle+b_{+}|6\rangle,
\end{gathered}
$$

with $a_{ \pm}$and $b_{ \pm}$complex quantities satisfying $\left|a_{ \pm}\right|^{2}+\left|b_{ \pm}\right|^{2}$ $=1$ and defined as

$$
\begin{gathered}
a_{-} \equiv \frac{\Omega}{\sqrt{\left|\zeta_{-}\right|^{2}+\Omega^{2}}}, \quad b_{-} \equiv \frac{\zeta_{-}}{\sqrt{\left|\zeta_{-}\right|^{2}+\Omega^{2}}}, \\
a_{+} \equiv-\frac{\Omega}{\Delta+i \gamma} \sqrt{\frac{\Delta^{2}+\gamma^{2}}{\left|\zeta_{+}\right|^{2}+\Omega^{2}}}, \\
b_{+} \equiv-\frac{\zeta_{+}}{\Delta+i \gamma} \sqrt{\frac{\Delta^{2}+\gamma^{2}}{\left|\zeta_{+}\right|^{2}+\Omega^{2}}}, \\
\zeta_{ \pm} \equiv-\frac{(\Delta+i \gamma)}{2} \pm \frac{1}{2}\left[\sqrt{\frac{|Z|+\operatorname{Re}(Z)}{2}}-i \sqrt{\frac{|Z|-\operatorname{Re}(Z)}{2}}\right], \\
Z \equiv(\Delta+i \gamma)^{2}+4 \Omega^{2}=\left(\Delta^{2}+4 \Omega^{2}-\gamma^{2}\right)+2 i \gamma \Delta .
\end{gathered}
$$

Although $\left|D_{1}\right\rangle$ and $\left|D_{3}\right\rangle$ are normalized and linearly independent, they are not orthogonal. The same occurs with $\left|D_{2}\right\rangle$ and $\left|D_{6}\right\rangle$. It is also worth noticing that $\Delta<0$ and $|\Delta|>\gamma$ $>0$, as we assume in the following.

It is easy to show that the quantities $a_{ \pm}=a_{ \pm}(\Delta, \Omega, \gamma)$ and $b_{ \pm}=b_{ \pm}(\Delta, \Omega, \gamma)$ satisfy the property $a_{ \pm}(\lambda \Delta, \lambda \Omega, \lambda \gamma)$ $=a_{ \pm}(\Delta, \Omega, \gamma)$ and $b_{ \pm}(\lambda \Delta, \lambda \Omega, \lambda \gamma)=b_{ \pm}(\Delta, \Omega, \gamma)$ for all real $\lambda>0$. Hence, by choosing $\lambda=1 /|\vec{\Delta}|$, we obtain $a_{ \pm}$ $=a_{ \pm}(-1, \Omega /|\Delta|, \gamma /|\Delta|)$ and $b_{ \pm}=b_{ \pm}(-1, \Omega /|\Delta|, \gamma /|\Delta|)^{-}$, that is, $a_{ \pm}$and $b_{ \pm}$can be viewed as functions of the two independent variables $\Omega /|\Delta|$ and $\gamma /|\Delta|$. The sign of $\Delta$ is fixed, $\Delta<0$, thus $a_{ \pm}$and $b_{ \pm}$are fully specified by giving $\Omega /|\Delta|$ and $\gamma /|\Delta|$. In this paper we are interested in the case of large red detunings, namely, $|\Delta| \gg \gamma$ and $|\Delta| \gg \Omega$. In particular, we can expand $a_{ \pm}$and $b_{ \pm}$up to the second order in $\Omega /|\Delta|$ to obtain

$$
\begin{gathered}
a_{-} \approx 1-\frac{\Omega^{2}}{2\left(\Delta^{2}+\gamma^{2}\right)}, \quad b_{-} \approx \frac{\Omega}{\Delta+i \gamma}, \\
a_{+} \approx-\frac{\Omega}{\Delta+i \gamma}, \quad b_{+} \approx 1-\frac{\Omega^{2}}{2\left(\Delta^{2}+\gamma^{2}\right)}\left[1+\frac{4 i \gamma \Delta}{\Delta^{2}+\gamma^{2}}\right]
\end{gathered}
$$

The scattering boundary conditions are more naturally stated in the dressed-state representation, Eqs. (14) and (15), than in the undressed, Eqs. (1), as we show below. Therefore, if we define the $6 \times 1$ column matrix $G_{\beta}(R)$ $\equiv M^{-1} F_{\beta}(R)$, where $M$ is the $6 \times 6$ complex matrix defined as

$$
M \equiv\left[\begin{array}{cccccc}
a_{-} & 0 & a_{+} & 0 & 0 & 0 \\
0 & a_{-} & 0 & 0 & 0 & a_{+} \\
b_{-} & 0 & b_{+} & 0 & 0 & 0 \\
0 & 0 & 0 & 1 & 0 & 0 \\
0 & 0 & 0 & 0 & 1 & 0 \\
0 & b_{-} & 0 & 0 & 0 & b_{+}
\end{array}\right]
$$


then Eq. (11) is equivalently written in terms of the dressed states and the radial amplitude $6 \times 1$ column matrix $G_{\beta}(R)$ as

$$
\left|\Psi_{\beta}(\mathbf{R})\right\rangle=\sum_{\sigma=1}^{6} \frac{F_{\sigma, \beta}(R)}{R}|\sigma\rangle=\sum_{\sigma=1}^{6} \frac{G_{\sigma, \beta}(R)}{R}\left|D_{\sigma}\right\rangle,
$$

where $\quad G_{\sigma, \beta}(R)=\Sigma_{\nu=1}^{6}\left(M^{-1}\right)_{\sigma, \nu} F_{\nu, \beta}(R) . \quad$ By $\quad$ leftmultiplying Eq. (12) by $M^{-1}$ and noticing that $V(R) F_{\beta}(R)$ $=V(R) M M^{-1} F_{\beta}(R)=V(R) M G_{\beta}(R)$, we obtain the matrix Schrödinger equation in the dressed-state representation:

$$
\begin{aligned}
& \frac{d^{2}}{d R^{2}} G_{\beta}(R)-\frac{2 \mu}{\hbar^{2}} M^{-1} V_{\infty} M G_{\beta}(R)-\frac{\mathbf{L}^{2}}{\hbar^{2} R^{2}} G_{\beta}(R) \\
& \quad-U(R) G_{\beta}(R)=0,
\end{aligned}
$$

where we have added and subtracted $M^{-1} V_{\infty} M$ and introduced the $6 \times 6$ complex matrix $U(R)$, satisfying $\lim _{R \rightarrow \infty} U(R)=0$ and $U\left(R \approx R_{\infty}\right) \approx 0$, and defined in terms of the potential matrix $V(R)$ as

$$
U(R) \equiv \frac{2 \mu}{\hbar^{2}} M^{-1}\left[V(R)-\mathbf{1}_{6} E-V_{\infty}\right] M,
$$

with $\mathbf{1}_{6}$ representing the $6 \times 6$ identity matrix. Let us also notice that $\mathbf{L}^{2}$ satisfies the property $M^{-1} \mathbf{L}^{2} M=\mathbf{L}^{2}$, since the radiation does not couple states of different rotational quantum numbers [32].

The states $\left|D_{1}\right\rangle$ and $\left|D_{2}\right\rangle$ have degenerate eigenvalues $-E+\hbar \zeta_{-}$of $V_{\infty}$, the states $\left|D_{3}\right\rangle$ and $\left|D_{6}\right\rangle$ have degenerate eigenvalues $-E+\hbar \zeta_{+}$, and the states $\left|D_{4}\right\rangle$ and $\left|D_{5}\right\rangle$ have degenerate eigenvalues $-E-\hbar \Delta_{c}$. In this article we assume that the kinetic energy $E_{k} \equiv \hbar^{2} k^{2} /(2 \mu)>0$ is much less than $-\hbar \Delta>0$, since we are considering the regime of ultracold temperatures and large red detunings. Asymptotically, at $t$ $=0$, let us suppose we prepare the system in the state $\left|D_{\alpha}\right\rangle$, for $\alpha=1,2$; the total Hamiltonian eigenvalue of the system, if the atoms are fixed at rest at position $R_{\infty}$, is equal to $\hbar \zeta_{-}$, because then $\left(d^{2} / d R^{2}\right) G_{\alpha, \beta}(R)=0$. Therefore, if we prepare the system in the state $\left|D_{\alpha}\right\rangle$, for $\alpha=1,2$, but now with a finite kinetic energy $E_{k}$, then $\left(d^{2} / d R^{2}\right) G_{\alpha, \beta}(R)=$ $-2 \mu E_{k} / \hbar^{2} G_{\alpha, \beta}(R)$ and $E=E_{k}+\hbar \zeta_{-}=\hbar^{2} k^{2} /(2 \mu)+\hbar \zeta_{-}$. It follows from Eqs. (15c) and (15d) that the imaginary part of the eigenvalue $E$ is $\operatorname{Im}\left(\hbar \zeta_{-}\right)<0$ and that $\operatorname{Im}\left(\hbar \zeta_{-}\right)$van- ishes as $|\Delta|$ becomes much greater than $\Omega$. Moreover, choosing a positive real kinetic energy is equivalent to imposing oscillatory asymptotic solutions for $G_{\alpha, \beta}(R)$, that is, $G_{\alpha, \beta}(R) \approx c_{1} \exp [i k R]+c_{2} \exp [-i k R]$, with $c_{1}$ and $c_{2}$ being independent of $R$ for $R \approx R_{\infty}$. Now, for example, for $\rho$ $=3,6$ and using $E=E_{k}+\hbar \zeta_{-}$, we have the asymptotic radial equation $d^{2} G_{\rho, \beta}(R) / d R^{2}-\Upsilon G_{\rho, \beta}(R)=0$, with the definition $\mathrm{Y} \equiv 2 \mu\left(-E_{k}-\hbar \zeta_{-}+\hbar \zeta_{+}\right) / \hbar^{2}$. The function $G_{\rho, \beta}(R)$ assumes the asymptotic behavior $G_{\rho, \beta}(R) \approx d_{1} \exp \left[v_{r} R\right.$ $\left.+i v_{i} R\right]+d_{2} \exp \left[-v_{r} R-i v_{i} R\right]$, where $d_{1}$ and $d_{2}$ are independent of $R$ for $R \approx R_{\infty}, \quad v_{r} \equiv \sqrt{[|\mathrm{Y}|+\operatorname{Re}(Y)] / 2}$, and $v_{i} \equiv$ $-\sqrt{[|Y|-\operatorname{Re}(Y)] / 2}\left[\sqrt{\Upsilon}= \pm\left(v_{r}+i v_{i}\right)\right.$, since it follows from Eqs. $(15 \mathrm{c})$ and $(15 \mathrm{~d})$ that $\operatorname{Im}(Y)<0]$. Because $v_{r}>0$ we must impose $d_{1}=0$ for $G_{\rho, \beta}(R)$ to be finite asymptotically; indeed, with $d_{1}=0, G_{\rho, \beta}(R)$ vanishes for $R \approx R_{\infty}$. A similar reasoning implies that $G_{\rho, \beta}(R)$, for $\rho=4,5$, also vanishes for $R \approx R_{\infty}$. Thus only the states $\left|D_{1}\right\rangle$ and $\left|D_{2}\right\rangle$ are accessible asymptotically, corresponding to open channels for the collision; the other states are therefore the closed channels in this problem, manifesting themselves only through resonances.

In the dressed-state representation the off-diagonal matrix elements of the Hamiltonian vanish asymptotically and we can apply the analysis of Ref. [16] generalized to the multichannel case. The first step is then to distinguish between closed and open channels and break Eq. (18) into two matrix equations, one for the open-channel manifold and the other for the closed-channel manifold. As we have discussed in the previous paragraph, the open-channel manifold corresponds to the $2 \times 2$ block of the Hamiltonian comprising the subspace spanned by the first two dressed states $\left|D_{1}\right\rangle$ and $\left|D_{2}\right\rangle$. The closed-channel manifold is spanned by the last four dressed states, corresponding to a $4 \times 4$ block of the Hamiltonian. The open-channel $2 \times 2$ block is coupled to the closed-channel $4 \times 4$ block by the off-diagonal blocks: one $2 \times 4$ and the other $4 \times 2$. Thus, we can write the $6 \times 6$ matrix $U(R)$ as a $2 \times 2$ matrix whose elements are also matrices:

$$
U(R)=\left[\begin{array}{cc}
U_{g g}(R) & U_{g e}(R) \\
U_{e g}(R) & U_{e e}(R)
\end{array}\right],
$$

where $U_{g g}(R)$ is a $2 \times 2$ complex matrix, $U_{e e}(R)$ is $4 \times 4$, $U_{g e}(R)$ is $2 \times 4$, and $U_{e g}(R)$ is $4 \times 2$. Now we are able to give explicit expressions of the different blocks of the matrix $U(R)$, Eq. (20). From Eqs. (9), (13), (17), and (19), it follows that

$$
\begin{gathered}
U_{g g}(R)=\frac{2 \mu}{\Gamma \hbar^{2}}\left[\begin{array}{cc}
a_{-} b_{+} W_{0}^{0}(R)-a_{+} b_{-} V_{a}(R) & 2 \sqrt{5} a_{+} b_{-} D(R) / 15 \\
2 \sqrt{5} a_{+} b_{-} D(R) / 15 & a_{-} b_{+} W_{0}^{0}(R)-a_{+} b_{-} V_{c}(R)
\end{array}\right], \\
U_{g e}(R)=\frac{2 \mu}{\Gamma \hbar^{2}}\left[\begin{array}{cccc}
a_{+} b_{+}\left[W_{0}^{0}(R)-V_{a}(R)\right] & -a_{+} D(R) / \sqrt{15} & -a_{+} D(R) / \sqrt{15} & 2 \sqrt{5} a_{+} b_{+} D(R) / 15 \\
2 \sqrt{5} a_{+} b_{+} D(R) / 15 & -a_{+} \sqrt{3} D(R) / 21 & -a_{+} \sqrt{3} D(R) / 21 & a_{+} b_{+}\left[W_{0}^{0}(R)-V_{c}(R)\right]
\end{array}\right],
\end{gathered}
$$




$$
U_{e g}(R)=\frac{2 \mu}{\Gamma \hbar^{2}}\left[\begin{array}{cc}
-a_{-} b_{-}\left[W_{0}^{0}(R)-V_{a}(R)\right] & -2 \sqrt{5} a_{-} b_{-} D(R) / 15 \\
b_{-} D(R) / \sqrt{15} & \sqrt{3} b_{-} D(R) / 21 \\
b_{-} D(R) / \sqrt{15} & \sqrt{3} b_{-} D(R) / 21 \\
-2 \sqrt{5} a_{-} b_{-} D(R) / 15 & -a_{-} b_{-}\left[W_{0}^{0}(R)-V_{c}(R)\right]
\end{array}\right]
$$

and, finally,

$$
U_{e e}(R)=\frac{2 \mu}{\Gamma \hbar^{2}}\left[\begin{array}{cccc}
a_{-} b_{+} V_{a}(R)-a_{+} b_{-} W_{0}^{0}(R) & a_{-} D(R) / \sqrt{15} & a_{-} D(R) / \sqrt{15} & -2 \sqrt{5} a_{-} b_{+} D(R) / 15 \\
b_{+} D(R) / \sqrt{15} & V_{b}(R) & -2 D(R) / 7 & \sqrt{3} b_{+} D(R) / 21 \\
b_{+} D(R) / \sqrt{15} & -2 D(R) / 7 & V_{b}(R) & \sqrt{3} b_{+} D(R) / 21 \\
-2 \sqrt{5} a_{-} b_{+} D(R) / 15 & \sqrt{3} a_{-} D(R) / 21 & \sqrt{3} a_{-} D(R) / 21 & a_{-} b_{+} V_{c}(R)-a_{+} b_{-} W_{0}^{0}(R)
\end{array}\right],
$$

where $\Gamma \equiv a_{-} b_{+}-a_{+} b_{-}$is the determinant of $M$. The $6 \times 6$ complex matrix $U(R)$ is not symmetric, reflecting the fact that the Hamiltonian is not Hermitian to account for spontaneous emission.

Let us define the $2 \times 1$ column matrix $G_{g \beta}(R) \equiv\left[G_{1, \beta}(R) G_{2, \beta}(R)\right]^{t}$ and the $4 \times 1$ column matrix $G_{e \beta}(R) \equiv\left[G_{3, \beta}(R)\right.$ $\left.G_{4, \beta}(R) G_{5, \beta}(R) G_{6, \beta}(R)\right]^{t}$. Thus, it is straightforward to derive from Eqs. (18) and (20) the following matrix equations:

$$
\begin{gathered}
\frac{d^{2}}{d R^{2}} G_{g \beta}(R)+\left[k^{2} \mathbf{1}_{2}-\frac{\mathbf{L}_{g g}^{2}}{\hbar^{2} R^{2}}-U_{g g}(R)\right] G_{g \beta}(R)=U_{g e}(R) G_{e \beta}(R), \\
\frac{d^{2}}{d R^{2}} G_{e \beta}(R)-\left[\kappa^{2} \mathbf{1}_{4}+s+\frac{\mathbf{L}_{e e}^{2}}{\hbar^{2} R^{2}}+U_{e e}(R)\right] G_{e \beta}(R)=U_{e g}(R) G_{g \beta}(R),
\end{gathered}
$$

where $k^{2} \equiv 2 \mu E_{k} / \hbar^{2} \equiv 2 \mu\left(E-\hbar \zeta_{-}\right) / \hbar^{2}, \kappa^{2} \equiv-2 \mu\left[E_{k}+\operatorname{Re}\left(\hbar \zeta_{-}-\hbar \zeta_{+}\right)\right] / \hbar^{2}, \mathbf{1}_{2}$ is the $2 \times 2$ identity matrix, $\mathbf{L}_{g g}^{2}$ is a $2 \times 2$ diagonal matrix containing the sequence $\left(0,6 \hbar^{2}\right)$ along its diagonal, $\mathbf{1}_{4}$ is the $4 \times 4$ identity matrix, $s$ is a $4 \times 4$ diagonal matrix defined as

$$
s \equiv-\frac{2 \mu}{\hbar}\left[\begin{array}{cccc}
i \operatorname{Im}\left(\zeta_{-}-\zeta_{+}\right) & 0 & 0 & 0 \\
0 & \operatorname{Re}\left(\zeta_{+}\right)+\Delta+i \gamma+i \operatorname{Im}\left(\zeta_{-}\right) & 0 & 0 \\
0 & 0 & \operatorname{Re}\left(\zeta_{+}\right)+\Delta+i \gamma+i \operatorname{Im}\left(\zeta_{-}\right) & 0 \\
0 & 0 & 0 & i \operatorname{Im}\left(\zeta_{-}-\zeta_{+}\right)
\end{array}\right],
$$

and $\mathbf{L}_{e e}^{2}$ is a $4 \times 4$ diagonal matrix containing the sequence $\left(0,6 \hbar^{2}, 6 \hbar^{2}, 6 \hbar^{2}\right)$ along its diagonal.

Equations (25) are in a form suitable for applying scattering boundary conditions, because $U_{g g}(R), U_{e e}(R), U_{g e}(R)$, and $U_{e g}(R)$ vanish asymptotically and so does the physically acceptable solution for the matrix $G_{e \beta}(R)$, as we have discussed above. As $R$ tends to $R_{\infty}$, it follows from Eq. (25a) that the asymptotic form of $G_{g \beta}(R)$ can be written as [33]

$$
G_{\alpha, \beta}(R) \approx i \sqrt{\frac{\mu}{2 \pi \hbar^{2} k}}\left\{\delta_{\alpha, \beta} \exp \left[-i\left(k R-\frac{\pi l_{\beta}}{2}\right)\right]-S_{\alpha, \beta} \exp \left[i\left(k R-\frac{\pi l_{\alpha}}{2}\right)\right]\right\},
$$

where $\alpha, \beta=1,2, l_{1} \equiv 0$ ( $s$ wave) and $l_{2} \equiv 2$ ( $d$ wave), and $S_{\alpha, \beta}$ are the elements of the scattering matrix. From elementary collision theory the meaning of the index $\beta$ is now clear: $\beta=1$ corresponds to an $s$-wave in the entrance channel, while $\beta=2$ corresponds to a $d$-wave. Analogously, the index $\alpha$ specifies the exit-channel $s$ - or $d$-wave. In the following sections we establish an algorithm to obtain the $2 \times 2 S$ matrix for this problem.

\section{THE CLOSED-CHANNEL MANIFOLD}

The $4 \times 4$ matrix $U_{e e}(R)$ appearing in Eq. (25b) can be viewed as a function not only of $R$, but also of the parameters $a_{ \pm}$and $b_{ \pm}$defined by Eqs. (15). Thus, we can write, explicitly, $U_{e e}(R) \equiv U_{e e}\left(a_{-}, b_{-}, a_{+}, b_{+}, R\right)$. It is apparent from their definition and Eqs. (16) that $a_{-}$and $b_{+}$tend to 1 , and $b_{-}$and $a_{+}$to 0 in the limit of vanishing $\Omega$ or infinite $|\Delta|$. Taking the case of zero intensity or infinite red detuning as reference, we define the $4 \times 4$ matrices $U_{0}(R) \equiv U_{e e}\left(a_{-}\right.$ $\left.=1, b_{-}=0, a_{+}=0, b_{+}=1, R\right) \quad$ and $\quad U_{p}(R) \equiv U_{p}\left(a_{-}, b_{-}\right.$, $\left.a_{+}, b_{+}, R\right) \equiv U_{e e}\left(a_{-}, b_{-}, a_{+}, b_{+}, R\right)-U_{0}(R)$. Explicit expressions for $U_{0}(R)$ and $U_{p}(R)$ are readily obtained from Eq. (24) and we notice that $U_{0}(R)$ is a real $4 \times 4$ matrix. The eigenvalues of $U_{0}(R)$ are $2 \mu W_{0}^{1}(R) / \hbar^{2}, 2 \mu W_{1}^{1}(R) / \hbar^{2}$ (doubly degenerate), and $2 \mu\left[3 W_{0}^{1}(R) / 7+4 W_{1}^{1}(R) / 7\right] / \hbar^{2}$. Let $d_{0}(R)$ be the $4 \times 4$ diagonal matrix obtained by diagonalizing $U_{0}(R)$. It is easy to verify that there is a $4 \times 4$ orthogonal matrix $A\left(A^{-1}=A^{t}\right)$, independent of $R$, such that $d_{0}(R)$ 
$=A^{t} U_{0}(R) A$. An explicit form of $A$ can be chosen as

$$
A=\left[\begin{array}{cccc}
\sqrt{3} / 3 & 0 & \sqrt{6} / 3 & 0 \\
-\sqrt{5} / 5 & \sqrt{2} / 2 & \sqrt{10} / 10 & \sqrt{5} / 5 \\
-\sqrt{5} / 5 & -\sqrt{2} / 2 & \sqrt{10} / 10 & \sqrt{5} / 5 \\
2 \sqrt{15} / 15 & 0 & -\sqrt{30} / 15 & \sqrt{15} / 5
\end{array}\right],
$$

whose columns give orthonormal eigenvectors of $U_{0}(R)$. Hence, writing $U_{e e}(R)=U_{0}(R)+U_{p}(R)$ in Eq. (25b) and left-multiplying the resulting equation by $A^{t}$, we obtain, after rearranging terms and defining $\widetilde{G}_{e \beta}(R) \equiv A^{t} G_{e \beta}(R)$,

$$
\begin{aligned}
\frac{d^{2}}{d R^{2}} & \widetilde{G}_{e \beta}(R)-\left[\kappa^{2} \mathbf{1}_{4}+d_{0}(R)\right] \widetilde{G}_{e \beta}(R) \\
& =A^{t} U_{e g}(R) G_{g \beta}(R)+A^{t} u_{e e}(R) G_{e \beta}(R),
\end{aligned}
$$

where, for convenience, we have defined the $4 \times 4$ complex matrix $u_{e e}(R)$ as

$$
u_{e e}(R) \equiv \frac{\mathbf{L}_{e e}^{2}}{\hbar^{2} R^{2}}+U_{p}(R)+s .
$$

Following the procedure of Ref. [16], now we solve Eq. (29) in terms of its Green function, using its right-hand side as the source. Accordingly, let $\phi_{n}(R)$ be the $4 \times 1$ column matrix satisfying the homogeneous differential equation $d^{2} \phi_{n}(R) / d R^{2}-d_{0}(R) \phi_{n}(R)=\kappa_{n}^{2} \phi_{n}(R)$, where $\kappa_{n}^{2}$ is a $4 \times 4$ diagonal matrix containing the sequence of positive numbers $\left(\kappa_{n, 3}^{2}, \kappa_{n, 4}^{2}, \kappa_{n, 5}^{2}, \kappa_{n, 6}^{2}\right)$ along its diagonal (in the closedchannel manifold the indices run from 3 to 6). The index $n$ $=1,2,3, \ldots$ enumerates the discrete spectrum of this homogeneous differential equation. Because $d_{0}(R)$ is real, each element of $\phi_{n}(R)$ can be chosen as a real function. The homogeneous problem has, besides the discrete, also a continuous spectrum. However, as we have discussed in the previous section, we are looking for solutions such that $G_{e \beta}(R)$ vanishes asymptotically and, therefore, we construct the corresponding Green function in terms of the discrete eigenfunctions only. Since the functions $\phi_{n}(R)$ for all natural numbers $n$ form a complete set in the discrete spectrum of the homogeneous problem, the solution of Eq. (29) can be expressed as

$$
\widetilde{G}_{\nu, \beta}(R)=\sum_{n} \frac{g_{n, \nu, \beta}}{\kappa_{n, \nu}^{2}-\kappa^{2}} \phi_{n, \nu}(R),
$$

where the index $\nu$ assumes the values from 3 to 6 to specify the 4 matrix elements of $\widetilde{G}_{e \beta}(R)$ and $\phi_{n}(R)$, and the quantities $g_{n, \nu, \beta}$ involve the source terms on the right-hand side of Eq. (29):

$$
\begin{aligned}
g_{n, \nu, \beta}= & \int_{0}^{R_{\infty}} \phi_{n, \nu}(R)\left[A^{t} U_{e g}(R) G_{g \beta}(R)\right. \\
& \left.+A^{t} u_{e e}(R) G_{e \beta}(R)\right]_{\nu} d R .
\end{aligned}
$$

Equations (31) and (32) are easily verified by substitution into Eq. (29) and assuming that $\phi_{n, \nu}(R)$ are properly nor- malized. In the notation of Eq. (32), a symbol like $[Q]_{\nu}$ stands for the element with index $\nu$ of the $4 \times 1$ column matrix $Q$. Let us notice that $\kappa_{n, 4}^{2}=\kappa_{n, 5}^{2}$ and $\phi_{n, 4}(R)$ $=\phi_{n, 5}(R)$ for all $n$, since both pairs of quantities $\left[\kappa_{n, 4}^{2}, \phi_{n, 4}(R)\right]$ and $\left[\kappa_{n, 5}^{2}, \phi_{n, 5}(R)\right]$ correspond to the same eigenvalue of $U_{0}(R)$, namely, $2 \mu W_{1}^{1}(R) / \hbar$. Moreover, we also assume that there are values of $n$ and $\nu=3,4,5,6$ for which $\kappa_{n, \nu}^{2}=\phi_{n, \nu}(R)=0$. It is particularly true that a purely repulsive potential $W_{1}^{1}(R)$ does not support bound states and, in this case, surely $\kappa_{n, 4}^{2}=\kappa_{n, 5}^{2}=\phi_{n, 4}(R)=\phi_{n, 5}(R)=0$ for all $n ; \kappa_{n, 6}^{2}$ and $\phi_{n, 6}(R)$ are different from zero for some $n$ only if the eigenvalue $2 \mu\left[3 W_{0}^{1}(R) / 7+4 W_{1}^{1}(R) / 7\right] / \hbar^{2}$ of $U_{0}(R)$ supports bound states.

We assume that the light field is nearly resonant with an isolated bound state, say $n=r$, of the attractive $W_{0}^{1}(R)$ potential (of ${ }^{3} \Sigma_{g}^{+}$symmetry). Thus, $\kappa^{2} \approx \kappa_{r, 3}^{2}$, since $\nu=3$ is the index corresponding to the eigenstate of $U_{0}(R)$ with eigenvalue $2 \mu W_{0}^{1}(R) / \hbar^{2}$, as explained above. Now, $G_{\rho, \beta}(R)=\Sigma_{\nu=3}^{6} A_{\rho, \nu} \widetilde{G}_{\nu, \beta}(R)$ for $\rho=3,4,5,6$ and, from Eq. (31), we can approximate $G_{\rho, \beta}(R)$ by taking into account only the resonant term and neglecting the others:

$$
G_{\rho, \beta}(R) \approx A_{\rho, 3} \frac{g_{r, 3, \beta}}{\kappa_{r, 3}^{2}-\kappa^{2}} \phi_{r, 3}(R) .
$$

Let us notice that in Eq. (28) all the elements $A_{\rho, 3}$ are different from zero, showing that the dominant contribution to $G_{\rho, \beta}(R)$ is indeed given by Eq. (33) for all $\rho=3,4,5,6$. This resonant approximation was checked numerically for the two-state problem of Ref. [20] and turned out to be excellent. After some algebraic manipulation of Eqs. (32) and (33), we obtain

$$
G_{\rho, \beta}(R) \approx A_{\rho, 3} \frac{\Theta_{r, 3, \beta}}{\kappa_{r, 3}^{2}-\kappa^{2}-w_{r, 3}} \phi_{r, 3}(R),
$$

where

$$
\begin{aligned}
& \Theta_{r, 3, \beta} \equiv \int_{0}^{R_{\infty}} \phi_{r, 3}(R)\left[A^{t} U_{e g}(R) G_{g \beta}(R)\right]_{3} d R, \\
& w_{r, 3} \equiv \int_{0}^{R_{\infty}} \phi_{r, 3}(R)\left[A^{t} u_{e e}(R) A\right]_{3,3} \phi_{r, 3}(R) d R .
\end{aligned}
$$

The notation $\left[A^{t} u_{e e}(R) A\right]_{3,3}$ in Eq. (35b) stands for the entry of the $4 \times 4$ matrix $A^{t} u_{e e}(R) A$ with row and column indices both equal to 3 .

\section{THE CONTINUUM GROUND STATES AND THE $S$ MATRIX}

Having found a valid approximation to the solution of the closed-channel problem, Eqs. (34) and (35), in this section we consider the open-channel manifold and the obtaining of the corresponding $2 \times 2$ scattering matrix. Similarly to $U_{e e}(R)$, the $2 \times 2$ potential matrix $U_{g g}(R)$, Eq. (21), is a function also of the parameters $a_{ \pm}$and $b_{ \pm}$of Eqs. (15), $U_{g g}(R) \equiv U_{g g}\left(a_{-}, b_{-}, a_{+}, b_{+}, R\right)$. Since we are interested 
in finding the modification in the collision process caused by the light field, in the previous section we have assumed the case of $a_{-}=b_{+}=1$ and $b_{-}=a_{+}=0$ as reference. Accordingly, let us define the $2 \times 2$ matrices: $U_{\text {ref }}(R) \equiv U_{g g}\left(a_{-}\right.$ $\left.=1, b_{-}=0, a_{+}=0, b_{+}=1, R\right)$, that is diagonal, and $U_{m}(R) \equiv U_{m}\left(a_{-}, b_{-}, a_{+}, b_{+}, R\right) \equiv U_{g g}\left(a_{-}, b_{-}, a_{+}, b_{+}, R\right)$ $-U_{\text {ref }}(R)$. It is easy to obtain explicit expressions of the matrices $U_{\text {ref }}(R)$ and $U_{m}(R)$ from Eq. (21). With these definitions, we rewrite Eq. (25a) as

$$
\begin{aligned}
& \frac{d^{2}}{d R^{2}} G_{g \beta}(R)+\left[k^{2} \mathbf{1}_{2}-\frac{\mathbf{L}_{g g}^{2}}{\hbar^{2} R^{2}}-U_{\mathrm{ref}}(R)\right] G_{g \beta}(R) \\
& =U_{g e}(R) G_{e \beta}(R)+U_{m}(R) G_{g \beta}(R) .
\end{aligned}
$$

The solution of the homogeneous differential equation speci- fied by making $U_{g e}(R)=0$ and $U_{m}(R)=0$ on the right-hand side of Eq. (36) gives the phase shifts for the entrance $s$ and $d$ waves in the absence of radiation. Thus, let $G_{\alpha}^{0}(R), \alpha$ $=1,2$, be the real regular solution of the homogeneous problem satisfying the scattering boundary condition: $G_{\alpha}^{0}(R)$ $\approx \sin \left(k R+\eta_{\alpha}-\pi l_{\alpha} / 2\right)$ as $R$ tends to $R_{\infty}$. Here, $l_{1} \equiv 0$ and $l_{2}$ $\equiv 2$, and $\eta_{\alpha} \equiv \eta_{\alpha}(k)$ is the phase shift of the $l_{\alpha}$ wave in the absence of the light field. Now let us take $G_{\alpha}^{1}(R)$, the real irregular solution of the homogeneous problem that has the asymptotic behavior $G_{\alpha}^{1}(R) \approx \cos \left(k R+\eta_{\alpha}-\pi l_{\alpha} / 2\right)$, and define the complex function $G_{\alpha}^{c}(R) \equiv G_{\alpha}^{1}(R) / k+i G_{\alpha}^{0}(R) / k$ that behaves asymptotically as $G_{\alpha}^{c}(R) \approx \exp \left[i\left(k R+\eta_{\alpha}\right.\right.$ $\left.\left.-\pi l_{\alpha} / 2\right)\right] / k$. Therefore, imposing the scattering boundary conditions of Eq. (27), it is straightforward to verify that the solution of Eq. (36) is [16]

$$
G_{\alpha, \beta}(R)=2 \sqrt{\frac{\mu}{2 \pi \hbar^{2} k}} \delta_{\alpha, \beta} G_{\alpha}^{0}(R) \exp \left[i \eta_{\alpha}\right]+\int_{0}^{R_{\infty}} \mathcal{G}_{\alpha}\left(R, R^{\prime}\right)\left[U_{g e}\left(R^{\prime}\right) G_{e \beta}\left(R^{\prime}\right)+U_{m}\left(R^{\prime}\right) G_{g \beta}\left(R^{\prime}\right)\right]_{\alpha} d R^{\prime}
$$

where $\mathcal{G}_{\alpha}\left(R, R^{\prime}\right)$ is the Green function given by $\mathcal{G}_{\alpha}\left(R, R^{\prime}\right) \equiv-G_{\alpha}^{0}(R) G_{\alpha}^{c}\left(R^{\prime}\right) \theta\left(R^{\prime}-R\right)-G_{\alpha}^{c}(R) G_{\alpha}^{0}\left(R^{\prime}\right) \theta\left(R-R^{\prime}\right)$, with the step function $\theta\left(x-x^{\prime}\right)=1$ if $x>x^{\prime}$ and $\theta\left(x-x^{\prime}\right)=0$ otherwise, for all real $x$ and $x^{\prime}$. From imposing the scattering boundary conditions and using Eqs. (34) and (35a), it also follows that

$$
S_{\alpha, \beta} \exp \left[-i \eta_{\alpha}\right]=\delta_{\alpha, \beta} \exp \left[i \eta_{\alpha}\right]-i \sqrt{\frac{2 \pi \hbar^{2}}{\mu k}} I_{\alpha, \beta}
$$

where the quantity $I_{\alpha, \beta}$ is given by

$$
I_{\alpha, \beta}=\frac{\Lambda_{r, 3, \alpha}}{\kappa_{r, 3}^{2}-\kappa^{2}-w_{r, 3}} \int_{0}^{R_{\infty}} \phi_{r, 3}(R)\left[A^{t} U_{e g}(R) G_{g \beta}(R)\right]_{3} d R+\int_{0}^{R_{\infty}} G_{\alpha}^{0}(R)\left[U_{m}(R) G_{g \beta}(R)\right]_{\alpha} d R,
$$

and we have defined $\Lambda_{r, 3, \alpha}$ as

$$
\Lambda_{r, 3, \alpha} \equiv \int_{0}^{R_{\infty}} G_{\alpha}^{0}(R)\left[U_{g e}(R) A\right]_{\alpha, 3} \phi_{r, 3}(R) d R
$$

The parameters $b_{-}$and $a_{+}$, according to Eqs. (16), are approximately $b_{-} \approx \Omega /(\Delta+i \gamma)$ and $a_{+} \approx-\Omega /(\Delta+i \gamma)$ for large red detunings $(\Omega /|\Delta| \ll 1)$. Using Eqs. (16), (21), (22), and (23), it follows that $U_{e g}(R)$ and $U_{g e}(R)$ are proportional to $\Omega /|\Delta|$ and $U_{m}(R)$ is proportional to $\Omega^{2} / \Delta^{2}$, in the limit of large red detunings. From Eqs. (39) and (40) it then follows that $I_{\alpha, \beta}$ is proportional to $\Omega^{2} / \Delta^{2}$. Hence, we can iterate Eq. (37) to obtain a perturbation series for $G_{g \beta}(R)$ in the parameter $\Omega^{2} / \Delta^{2}$, using also Eqs. (34) and (35a). Substituting this estimate of $G_{g \beta}(R)$ into Eqs. (38) and (39) gives the approximated scattering matrix. In this paper we illustrate this procedure up to the second order in $\Omega /|\Delta|$. In this case, Eqs. (37) and (39) give

$$
I_{\alpha, \beta}=\sqrt{\frac{2 \mu}{\pi \hbar^{2} k}}\left[\frac{\Lambda_{r, 3, \alpha} \widetilde{\Lambda}_{r, 3, \beta}}{\kappa_{r, 3}^{2}-\kappa^{2}-w_{r, 3}}+\int_{0}^{R_{\infty}} G_{\alpha}^{0}(R)\left[U_{m}(R)\right]_{\alpha, \beta} G_{\beta}^{0}(R) d R\right] \exp \left[i \eta_{\beta}\right],
$$

where we have defined

$$
\widetilde{\Lambda_{r, 3, \beta}} \equiv \int_{0}^{R_{\infty}} G_{\beta}^{0}(R)\left[A^{t} U_{e g}(R)\right]_{3, \beta} \phi_{r, 3}(R) d R
$$

Using Eqs. (38) and (41) gives the $S$ matrix elements:

$$
S_{\alpha, \beta}=\left[\delta_{\alpha, \beta}-\frac{2 i \Lambda_{r, 3, \alpha} \widetilde{\Lambda}_{r, 3, \beta}}{k\left(\kappa_{r, 3}^{2}-\kappa^{2}-w_{r, 3}\right)}-\frac{2 i}{k} \int_{0}^{R_{\infty}} G_{\alpha}^{0}(R)\left[U_{m}(R)\right]_{\alpha, \beta} G_{\beta}^{0}(R) d R\right] \exp \left[i\left(\eta_{\alpha}+\eta_{\beta}\right)\right] .
$$


Using Eqs. (10), (16), (21)-(24), (26), (28), (30), (35b), (40), and (42), we can rewrite the matrix elements of Eq. (43) explicitly as

$$
\begin{gathered}
S_{1,1}=\left\{1-\frac{2 \pi i \Omega^{2}}{(\Delta+i \gamma)^{2}}\left[\frac{\left[Q_{r, 3,1}\right]^{2}}{3\left[\left(E_{k}-\hbar \Delta_{r}\right)+i \hbar \gamma\right]}-P_{a}\right]\right\} \exp \left[2 i \eta_{1}\right], \\
S_{1,2}=S_{2,1}=\frac{4 \pi i \Omega^{2}}{3 \sqrt{5}(\Delta+i \gamma)^{2}}\left[P_{D}-\frac{Q_{r, 3,1} Q_{r, 3,2}}{\left(E_{k}-\hbar \Delta_{r}\right)+i \hbar \gamma}\right] \exp \left[i\left(\eta_{1}+\eta_{2}\right)\right], \\
S_{2,2}=\left\{1-\frac{2 \pi i \Omega^{2}}{(\Delta+i \gamma)^{2}}\left[\frac{4\left[Q_{r, 3,2}\right]^{2}}{15\left[\left(E_{k}-\hbar \Delta_{r}\right)+i \hbar \gamma\right]}-P_{c}\right]\right\} \exp \left[2 i \eta_{2}\right],
\end{gathered}
$$

where we have defined the quantities

$$
\begin{gathered}
Q_{r, 3, \alpha} \equiv \sqrt{\frac{2 \mu}{\pi k \hbar^{2}}} \int_{0}^{R_{\infty}} G_{\alpha}^{0}(R)\left[W_{0}^{1}(R)-W_{0}^{0}(R)\right] \phi_{r, 3}(R) d R, \\
P_{a} \equiv \frac{2 \mu}{\pi k \hbar^{2}} \int_{0}^{R_{\infty}} G_{1}^{0}(R)\left[W_{0}^{0}(R)-V_{a}(R)\right] G_{1}^{0}(R) d R, \\
P_{D} \equiv \frac{2 \mu}{\pi k \hbar^{2}} \int_{0}^{R_{\infty}} G_{1}^{0}(R)\left[W_{1}^{1}(R)-W_{0}^{1}(R)\right] G_{2}^{0}(R) d R, \\
P_{c} \equiv \frac{2 \mu}{\pi k \hbar^{2}} \int_{0}^{R_{\infty}} G_{2}^{0}(R)\left[W_{0}^{0}(R)-V_{c}(R)\right] G_{2}^{0}(R) d R, \\
E_{r} \equiv \frac{\hbar^{2}}{2 \mu} \int_{0}^{R_{\infty}} \phi_{r, 3}(R) \frac{1}{\kappa_{r, 3}^{2}} \phi_{r, 3}(R) d R, \quad \text { and } \quad \hbar \Delta_{r} \equiv-\hbar \Delta-E_{r}+4 \epsilon .
\end{gathered}
$$

Equations (44) are valid up to the second order in $\Omega /|\Delta|$. These $S$ matrix elements contain information relevant in the context of photoassociation line shapes and modification of scattering lengths. If $\gamma=0$, this theory is Hermitian and the $S$ matrix is unitary, $S^{\dagger}=S^{-1}$, meaning that the total probability is conserved. Thus, in the case of $\gamma \neq 0$ the total probability is not conserved and this loss is proportional to the amount of probability flux going into spontaneous emission from the bound state, that is, the total probability loss is proportional to the photoassociation line shape. Then we can obtain the line shape for an $s$ wave in the entrance channel by taking the difference $\Xi_{s} \equiv 1-\left|S_{1,1}\right|^{2}-\left|S_{1,2}\right|^{2}$, and, for a $d$ wave, we define $\Xi_{d} \equiv 1$ $-\left|S_{2,2}\right|^{2}-\left|S_{1,2}\right|^{2}$. From Eqs. (44) it follows that, up to the second order in $\Omega /|\Delta|$,

$$
\begin{aligned}
& \Xi_{s} \approx \frac{8 \pi \gamma \Omega^{2}}{\left(\Delta^{2}+\gamma^{2}\right)^{2}}\left[\frac{\left[Q_{r, 3,1}\right]^{2}\left[2 \Delta\left(E_{k}-\hbar \Delta_{r}\right)+\hbar\left(\Delta^{2}-\gamma^{2}\right)\right]}{6\left[\left(E_{k}-\hbar \Delta_{r}\right)^{2}+(\hbar \gamma)^{2}\right]}-P_{a} \Delta\right], \\
& \Xi_{d} \approx \frac{8 \pi \gamma \Omega^{2}}{\left(\Delta^{2}+\gamma^{2}\right)^{2}}\left[\frac{2\left[Q_{r, 3,2}\right]^{2}\left[2 \Delta\left(E_{k}-\hbar \Delta_{r}\right)+\hbar\left(\Delta^{2}-\gamma^{2}\right)\right]}{15\left[\left(E_{k}-\hbar \Delta_{r}\right)^{2}+(\hbar \gamma)^{2}\right]}-P_{c} \Delta\right] .
\end{aligned}
$$

Let us notice that these line shapes are asymmetric and require the knowledge of the $k$-dependent integrals of Eqs. (45). We do not attempt to calculate these integrals in the present article, because here we focus only on the theoretical procedure to obtain the line shapes of Eqs. (46). In a forthcoming paper we will calculate the quantities $\left[Q_{r, 3,1}\right]^{2}, P_{a},\left[Q_{r, 3,2}\right]^{2}$, and $P_{c}$ numerically, and present a comparison of the present theory with a numerical close-coupling calculation, to check the accuracy of this semianalytic approach. In a forthcoming paper we will also investigate the dependence of the collision process on the polarization of the light field. The line shapes of Eqs. (46), due to their physical relevance, will be granted full attention in this separate paper. Therefore, it is not our purpose here to analyze thoroughly the line shapes above, but it is important to mention that they show the Wigner-threshold-law behavior for low entrance kinetic energies, since in this case the quantities $\left[Q_{r, 3,1}\right]^{2}$ and $\left[Q_{r, 3,2}\right]^{2}$ are proportional to $k^{2 l+1}[20]$. 
For low enough entrance kinetic energies, the dominant contribution to the collision process comes from the $s$ wave alone and we can show that in this case $\eta_{1} \approx-k A_{0}$, where $A_{0}$ is an energy-independent parameter called the scattering length. In the presence of light, the scattering length is modified according to Eq. (44a). Thus, by writing $S_{1,1} \equiv \exp [-2 i k A] \approx 1-2 i k A$, we obtain

$$
\begin{aligned}
A \approx & A_{0}+\frac{\pi \Omega^{2}}{k\left(\Delta^{2}+\gamma^{2}\right)^{2}}\left[\frac{\left[Q_{r, 3,1}\right]^{2}\left[\left(\Delta^{2}-\gamma^{2}\right)\left(E_{k}-\hbar \Delta_{r}\right)-2 \hbar \Delta \gamma^{2}\right]}{3\left[\left(E_{k}-\hbar \Delta_{r}\right)^{2}+(\hbar \gamma)^{2}\right]}-\left(\Delta^{2}-\gamma^{2}\right) P_{a}\right] \\
& -\frac{2 i \pi \gamma \Omega^{2}}{k\left(\Delta^{2}+\gamma^{2}\right)^{2}}\left[\frac{\left[Q_{r, 3,1}\right]^{2}\left[2 \Delta\left(E_{k}-\hbar \Delta_{r}\right)+\hbar\left(\Delta^{2}-\gamma^{2}\right)\right]}{6\left[\left(E_{k}-\hbar \Delta_{r}\right)^{2}+(\hbar \gamma)^{2}\right]}-P_{a} \Delta\right],
\end{aligned}
$$

where $A$ is the modified scattering length that now acquires an imaginary part, reflecting the absorption into an unstable bound-state resonance. We notice also that if $\gamma=0$ the imaginary part vanishes, as we would expect. The quantities $\left[Q_{r, 3,1}\right]^{2}$ and $P_{a}$ are proportional to $k$ at low enough kinetic energies, and this cancels the $k$ in the denominator of the second and third terms on the right-hand side of Eq. (47). As we have mentioned above, in a forthcoming paper we will concentrate on the line shapes of photoassociation. We will also focus on a numerical investigation of the modification of the scattering length by light, given the importance of this subject for experimental purposes.

The present article is to be viewed as a discussion of multichannel predictions expressed by Eqs. (46) and (47). It is worth remarking that these results are consequences of the solution of the Schrödinger equation using approaches to different stages of the theory already considered usual in the literature. However, not all of these usual approaches are incorporated into a single unified treatment, as we have presented here. The importance of the present procedure resides in this unification of techniques and the possibility of its extension to treat more realistic situations, as the introduction of fine structure, for example. Also, if the magnitude of the detuning is greater than the Rabi frequency, the perturbative iteration of Eqs. (37)-(39) can be easily extended to higher orders in the perturbation parameter, $\Omega /|\Delta|$, as long as the power broadening is much less than the separation of the adjacent bound-state resonances, that is, as long as each resonance remains isolated.

\section{VALIDITY OF THE THEORY}

Our first approximation involves the neglect of fine and hyperfine structures; also the magnitude of the red detuning is assumed to be very large as compared with the Rabi frequency. If we choose $\Delta /(2 \pi c) \approx-1.0 \mathrm{~cm}^{-1}$, we satisfy both requirements: fine and hyperfine structures are small compared with the magnitude of the attractive potential and a Rabi frequency corresponding to $1 \mathrm{~cm}^{-1}$ implies an intensity of $I \approx 2 \times 10^{5} \mathrm{~W} / \mathrm{cm}^{2}$, meaning that $\Omega /|\Delta| \ll 1$ for any reasonable value of laser intensity. Adjacent ${ }^{3} \Sigma_{g}^{+}$resonances for $\Delta /(2 \pi c) \approx-1.0 \mathrm{~cm}^{-1}$ are separated by about 0.14 $\mathrm{cm}^{-1}$. Thus, imposing $\Omega /(2 \pi c) \ll 0.14 \mathrm{~cm}^{-1}$ implies that the intensity must satisfy $I \ll 4.5 \times 10^{3} \mathrm{~W} / \mathrm{cm}^{2}$. Such an intensity is achievable experimentally, and in this circumstance power broadening is important, requiring changing the present theory to include effects of more than one resonance being excited. This generalization to overlapping resonances is not impossible to accomplish, but we can still apply this isolated-resonance theory for red detunings of magnitudes satisfying $|\Delta| /(2 \pi c) \gtrsim 10.0 \mathrm{~cm}^{-1}$, in which case the separation between adjacent resonances is about $1.0 \mathrm{~cm}^{-1}$, implying the condition $I \ll 2 \times 10^{5} \mathrm{~W} / \mathrm{cm}^{2}$.

A typical situation in a trap corresponds to temperatures from a few tens to a few hundreds of $\mu \mathrm{K}$, and densities from about $10^{10}$ to $10^{14} \mathrm{~cm}^{-3}$. Let us fix the density to about $10^{11}$ $\mathrm{cm}^{-3}$. Then we estimate a mean interatomic distance of about $4 \times 10^{4} a_{0}$, where $a_{0}$ is the Bohr radius. The longestrange Born-Oppenheimer potential curves appearing in Eq. (9), for example, are the ones corresponding asymptotically to one ground-state ${ }^{2} S$ atom and one first-excited-state ${ }^{2} P$ atom. These curves behave asymptotically as $C_{3} / R^{3}$, where $C_{3}$ is on the order of a few atomic units [34]. Thus, the $R$-dependent part of the potential matrix is about $3 \times 10^{-9}$ $\mathrm{cm}^{-1}$. Even at the low laser intensity of $1 \mathrm{~W} / \mathrm{cm}^{2}$, the Rabi frequency is about $2 \times 10^{-3} \mathrm{~cm}^{-1}$, or about $10^{5}$ times greater than the magnitude of the $R$-dependent part of the potential matrix, and $|\Delta| /(2 \pi c) \approx 1.0 \mathrm{~cm}^{-1}$ is $10^{8}$ times greater. The centrifugal terms appearing in Eq. (12), for instance, are either zero or $3 \hbar^{2} /\left(\mu R^{2}\right)$. At $R_{\infty} \approx 4 \times 10^{4} a_{0}, 3 \hbar^{2} /\left(h c \mu R_{\infty}^{2}\right)$ $\approx 2 \times 10^{-8} \mathrm{~cm}^{-1}$. Thus, we can safely choose $R_{\infty}$ $\approx 4 \times 10^{4} a_{0}$ as the asymptotic region of this theory.

It now remains to determine the minimum asymptotic kinetic energy for which this theory is valid. We mentioned at the beginning of Sec. III that the stationary states of the total Hamiltonian have an exponentially decreasing amplitude due to loss through spontaneous emission. Accordingly, for a colliding system to survive during the whole process, this decreasing amplitude must remain significant from the start to the end of the scattering encounter. Let $v$ be the initial and final relative speeds of the colliding partners (in this problem the open channels correspond to the same asymptotic kinetic energy). Thus, the time taken for the atoms to move from a distance about $R_{\infty}$ to their encounter region is approximately $R_{\infty} / v$, and from their breaking apart to their reaching the asymptotic region again is on the same order, $R_{\infty} / v$. The total duration of the collision process is about $2 R_{\infty} / v$. It follows from Sec. III that the amplitude of the asymptotic stationary states decays as $\exp \left[\operatorname{Im}\left(\hbar \zeta_{-}\right) t\right]$, with $\operatorname{Im}\left(\hbar \zeta_{-}\right)$ $<0$. At the end of the collision, this factor gives $\exp \left[2 \operatorname{Im}\left(\hbar \zeta_{-}\right) R_{\infty} / v\right]$ and we impose $2\left|\operatorname{Im}\left(\hbar \zeta_{-}\right)\right| R_{\infty} / v \ll 1$, or $v \gg 2\left|\operatorname{Im}\left(\hbar \zeta_{-}\right)\right| R_{\infty}$. Up to the second order in $\Omega /|\Delta|$, this condition becomes 


$$
v \gg 2 \gamma R_{\infty} \frac{\Omega^{2}}{\Delta^{2}} .
$$

Using $\gamma /(2 \pi) \approx 10 \mathrm{MHz},|\Delta| /(2 \pi c) \approx 1.0 \mathrm{~cm}^{-1}, R_{\infty} \approx 4$ $\times 10^{4} a_{0}$, and $\Omega /(2 \pi c) \approx 1.47966 \times 10^{-3} \sqrt{2 I} \mathrm{~cm}^{-1}$, we obtain $v / I \gg 0.12 \mathrm{~cm} / \mathrm{s}$, where the intensity $I$ is given in $\mathrm{W} / \mathrm{cm}^{2}$. Using the reduced mass of sodium, $\mu \approx 1.91 \times 10^{-23} \mathrm{~g}$, and defining the collision temperature as $T \equiv \mu v^{2} /\left(3 k_{B}\right)$, where $k_{B}$ is Boltzmann's constant, according to Eq. (48) $T$ must satisfy the inequality

$$
\frac{T}{I} \gg 6.6 \times 10^{-10} \mathrm{~K},
$$

a condition well satisfied in current experiments.

\section{CONCLUSION}

In this article we have developed a non-Hermitian multichannel procedure to treat binary collisions of ultracold atoms in the presence of a red-detuned laser field. We have obtained the scattering matrix elements up to the second order in the ratio between the Rabi frequency and the detuning, and showed that this approach is valid for a significant range of experimental conditions. The perturbation series can be easily extended to higher orders. As practical results of these calculations, we have presented the multichannel line shapes of photoassociation spectroscopy for the $s$ and $d$ wave in the entrance channel, and the light-modified scattering length for low but finite entrance kinetic energies. Although we have truncated the partial waves at the $l=2$ manifold, the inclusion of higher partial waves is straightforward, at least for this case with no fine or hyperfine structure.

In future work, we will be considering the line shapes of Eqs. (46) and the modification of scattering lengths, Eq. (47), in greater detail. We will investigate numerically these quantities for conditions relevant to actual experiments. The differences between linear and circular polarization effects will be examined and we expect them to be appreciable, as in the case of blue detuning [10], due to the inherently different topologies of the respective sets of adiabatic potential curves. Guided by the present model of ultracold collisions, we will develop a fully numerical close-coupling calculation to treat the scattering exactly. The exact numerical results will determine the boundaries of the parameter space within which this semianalytic theory is valid. We expect that these investigations will be relevant to the interpretation of many experimental results yet to appear.

\section{ACKNOWLEDGMENTS}

This work was supported by FAPESP (Fundação de Amparo à Pesquisa do Estado de São Paulo). The author is very grateful to Vanderlei S. Bagnato, Paul S. Julienne, and John Weiner for their kind hospitality and fruitful discussions. The author is also indebted to Jayme De Luca for a careful reading of the manuscript and helpful suggestions. This work has also been partially supported by Convênio FINEP/PRONEX Grant No. 41/96/0935/00.
[1] S. Bali, D. Hoffmann, and T. Walker, Europhys. Lett. 27, 273 (1994)

[2] L. Marcassa, S. Muniz, E. de Queiroz, S. Zilio, V. Bagnato, J. Weiner, P. S. Julienne, and K.-A. Suominen, Phys. Rev. Lett. 73, 1911 (1994).

[3] H. Katori and F. Shimizu, Phys. Rev. Lett. 73, 2555 (1994).

[4] L. Marcassa, R. Horowicz, S. Zilio, V. Bagnato, and J. Weiner, Phys. Rev. A 52, R913 (1995).

[5] M. Walhout, U. Sterr, C. Orzel, M. Hoogerland, and S. L. Rolston, Phys. Rev. Lett. 74, 506 (1995).

[6] C. D. Wallace, V. Sanchez-Villicana, T. P. Dinneen, and P. L. Gould, Phys. Rev. Lett. 74, 1087 (1995).

[7] S. C. Zilio, L. Marcassa, S. Muniz, R. Horowicz, V. Bagnato, R. Napolitano, J. Weiner, and P. S. Julienne, Phys. Rev. Lett. 76, 2033 (1996).

[8] S. R. Muniz, L. G. Marcassa, R. Napolitano, G. D. Telles, J. Weiner, S. C. Zilio, and V. S. Bagnato, Phys. Rev. A 55, 4407 (1997).

[9] K.-A. Suominen, M. J. Holland, K. Burnett, and P. S. Julienne, Phys. Rev. A 51, 1446 (1995).

[10] R. Napolitano, J. Weiner, and P. S. Julienne, Phys. Rev. A 55, 1191 (1997).

[11] V. A. Yurovsky and A. Ben-Reuven, Phys. Rev. A 55, 3772 (1997).

[12] P. O. Fedichev, Yu. Kagan, G. V. Shlyapnikov, and J. T. M. Walraven, Phys. Rev. Lett. 77, 2913 (1996).
[13] M. H. Anderson, J. R. Ensher, M. R. Matthews, C. E. Wieman, and E. A. Cornell, Science 269, 198 (1995).

[14] C. C. Bradley, C. A. Sackett, J. J. Tollett, and R. G. Hulet, Phys. Rev. Lett. 75, 1687 (1995).

[15] K. B. Davis, M.-O. Mewes, M. R. Andrews, N. J. van Druten, D. S. Durfee, D. M. Kurn, and W. Ketterle, Phys. Rev. Lett. 75, 3969 (1995)

[16] R. Napolitano, Braz. J. Phys. 27, 162 (1997).

[17] P. S. Julienne and F. H. Mies, Phys. Rev. A 25, 3399 (1982).

[18] H. R. Thorsheim, J. Weiner, and P. S. Julienne, Phys. Rev. Lett. 58, 2420 (1987); V. Bagnato, L. Marcassa, Y. Wang, J. Weiner, P. S. Julienne, and Y. B. Band, Phys. Rev. A 48, R2523 (1993); V. Bagnato, L. Marcassa, C. Tsao, Y. Wang, and J. Weiner, Phys. Rev. Lett. 70, 3225 (1993); C.-C. Tsao, R. Napolitano, Y. Wang, and J. Weiner, Phys. Rev. A 51, R18 (1995); D. Leonhardt and J. Weiner, ibid. 52, R4332 (1995).

[19] P. D. Lett, K. Helmerson, W. D. Phillips, L. P. Ratliff, S. L. Rolston, and M. E. Wagshul, Phys. Rev. Lett. 71, 2200 (1993); M. E. Wagshul, K. Helmerson, and P. D. Lett, ibid. 70, 2074 (1993); L. P. Ratliff, M. E. Wagshul, P. D. Lett, S. L. Rolston, and W. D. Phillips, J. Chem. Phys. 101, 2638 (1994); P. D. Lett, P. S. Julienne, and W. D. Phillips, Annu. Rev. Phys. Chem. 46, 423 (1995); K. M. Jones, P. S. Julienne, P. D. Lett, W. D. Phillips, E. Tiesinga, and C. J. Williams, Europhys. Lett. 35, 85 (1996); K. M. Jones, S. Maleki, and P. D. Lett, J. Phys. B 30, 289 (1997). 
[20] R. Napolitano, J. Weiner, C. J. Williams, and P. S. Jullienne, Phys. Rev. Lett. 73, 1352 (1994).

[21] C. J. Williams and P. S. Julienne, J. Chem. Phys. 101, 2634 (1994); Y. B. Band and P. S. Julienne, Phys. Rev. A 51, R4317 (1995); J. L. Bohn and P. S. Julienne, ibid. 54, R4637 (1996).

[22] J. D. Miller, R. A. Cline, and D. J. Heinzen, Phys. Rev. Lett. 71, 2204 (1993); R. A. Cline, J. D. Miller, and D. J. Heinzen, ibid. 73, 632 (1994); H. M. J. M. Boesten, C. C. Tsai, B. J. Verhaar, and D. J. Heinzen, ibid. 77, 5194 (1996).

[23] W. I. McAlexander, E. R. I. Abraham, N. W. M. Ritchie, C. J. Williams, H. T. C. Stoof, and R. G. Hulet, Phys. Rev. A 51, R871 (1995); E. R. I. Abraham, W. I. McAlexander, C. A. Sackett, and R. G. Hulet, Phys. Rev. Lett. 74, 1315 (1995); E. R. I. Abraham, W. I. McAlexander, J. M. Gerton, R. G. Hulet, R. Coté, and A. Dalgarno, Phys. Rev. A 55, R3299 (1997).

[24] M. Machholm, A. Giusti-Suzor, and F. H. Mies, Phys. Rev. A 50, 5025 (1994); O. Dulieu, B. Lévy, S. Magnier, F. MasnouSeeuws, and Ph. Millié, Phys. Rev. Lett. 76, 2858 (1996); P. Pilet, A. Crubellier, and F. Masnou-Seeuws, J. Phys. B 30, 2801 (1997).
[25] P. A. Moelnaar, P. van der Straten, and H. G. M. Heideman, Phys. Rev. Lett. 77, 1460 (1996).

[26] H. Wang, J. Li, X. T. Wang, C. J. Williams, P. L. Gould, and W. C. Stwalley, Phys. Rev. A 55, R1569 (1997).

[27] H. T. C. Stoof, J. M. V. A. Koelman, and B. J. Verhaar, Phys. Rev. B 38, 4688 (1988).

[28] E. A. Power, J. Chem. Phys. 46, 4297 (1967).

[29] P. S. Julienne, K.-A. Suominen, and Y. Band, Phys. Rev. A 49, 3890 (1994); R. Solts, A. Ben-Reuven, and P. S. Julienne, ibid. 52, 4029 (1995).

[30] A. Messiah, Quantum Mechanics (North-Holland, Amsterdam, 1965).

[31] J. M. Launay, J. Phys. B 10, 3665 (1977).

[32] P. S. Julienne and F. H. Mies, Phys. Rev. A 30, 831 (1984).

[33] F. H. Mies, Phys. Rev. A 7, 942 (1973); Mol. Phys. 41, 953 (1980); Theoretical Chemistry: Advances and Perspectives, edited by D. Henderson (Academic Press, New York, 1981), Vol. 6B, p. 127.

[34] B. Bussery and M. Aubert-Frécon, J. Chem. Phys. 82, 3224 (1985). 